\title{
Phase Diagram of the quadrumerized Shastry-Sutherland Model
}

\author{
Andreas Läuchli, Stefan Wessel, and Manfred Sigrist \\ Institut für Theoretische Physik, ETH-Hönggerberg, CH-8093 Zürich, Switzerland
}

(November 7, 2018)

\begin{abstract}
We determine the phase diagram of a generalized Shastry-Sutherland model, using a combination of dimer- and quadrumer-boson methods and numerical exact diagonalization techniques. Along special lines in the parameter space the model reduces to the standard Shastry-Sutherland model, the $1 / 5$-th depleted square lattice and the two-dimensional plaquette square lattice model. We study the evolution of the ordered phases found in the latter two unfrustrated models under the effect of frustration. Furthermore we present new exact diagonalization results for the Shastry-Sutherland model on clusters with up to 32 sites, supporting the existence of an intermediate gapped valence bond crystal phase with plaquette long-ranged order.
\end{abstract}

There has been considerable interest in recent years in the study of low-dimensional quantum spin systems, both experimentally and theoretically. Special attention has been devoted to two-dimensional antiferromagnetic systems, where quantum fluctuations and frustration allow for various exotic quantum phases to compete with quasi-classical long-range magnetic order. [1]

Already in the unfrustrated regime such spin liquid phases occur in certain regions of the parameter space, a prominent example being the spin- $1 / 2$ Heisenberg model on the $1 / 5$-th depleted square lattice. [2] In this model two distinct spin liquid phases, well described by resonating valence bond (RVB) -like states, are found along with an intermediate long-ranged antiferromagnetically ordered phase. [2,34 The location of the quantum critical points separating these phases are known to rather high precision. 顿

When considering frustrated systems, ground state properties are less well established. A prominent example of recent interest, due to its relevance for the spin gap sys-

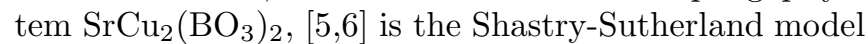
(SSM). 77 In this model the nearest-neighbor square lattice antiferromagnet is frustrated by additional diagonal interactions, arranged in a staggered pattern on alternate squares. This model retains long-range Néel-order for small diagonal coupling. Furthermore, it becomes an exact dimer valence bond solid (VBS) with singlets forming on the diagonal bonds for small axial coupling. Concerning the existence and nature of intermediate phases, despite numerous investigations, [8-12], a definite picture has not yet emerged.

\section{THE MODEL}

In this paper, we present exact diagonalization studies on the SSM that indicate the occurrence of a valence bond crystal (VBC) in the intermediate regime, with plaquette long-range order. Furthermore, we are able to link this phase to a consistent phase diagram of an extension of the SSM. Therefore, we introduce the quadrumerized $\mathrm{SSM}$, defined on the square lattice by the following spin1/2 Heisenberg Hamiltonian

$$
H=K \sum_{\langle i, j\rangle_{\square}} \mathbf{S}_{i} \cdot \mathbf{S}_{j}+J \sum_{\langle i, j\rangle_{\square}} \mathbf{S}_{i} \cdot \mathbf{S}_{j}+J^{\prime} \sum_{\langle\langle i, j\rangle\rangle} \mathbf{S}_{i} \cdot \mathbf{S}_{j} .
$$

Here $K$ and $J$ are the two inequivalent nearest neighbour exchange couplings, whereas $J^{\prime}$ denotes the next nearest neighbour alternating dimer coupling. The various couplings are displayed in Fig. 1.

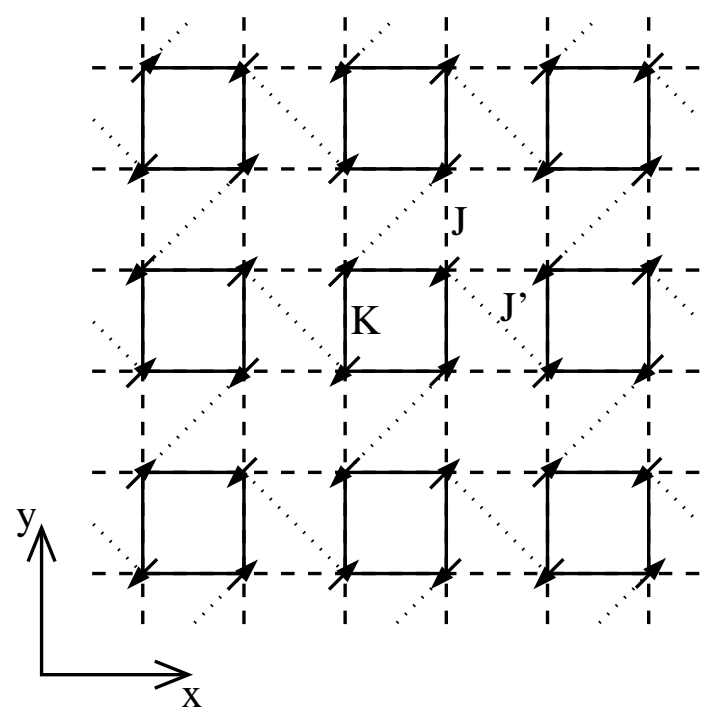

FIG. 1. The quadrumerized Shastry-Sutherland lattice. A spin-1/2 degree of freedom is located on each vertex. The various couplings, represented by different line styles, are denoted $K$ (solid), $J$ (dashed), and $J^{\prime}$ (dotted). The lattice is self-dual under the exchange $(J \leftrightarrow K)$. Arrows illustrate the ordered phase found for $J=0$ in the region $J^{\prime} \approx K$.

Note, that the lattice is self-dual under the exchange $(J \leftrightarrow K)$, hence only the case $J \leq K$ will be considered. Furthermore, the standard Shastry-Sutherland model is recovered along $J=K$, and has a larger space group symmetry. Other lines of enhanced symmetry correspond 
to the $1 / 5$-th depleted square lattice $(J=0)$, and the plaquette square lattice $\left(J^{\prime}=0\right)$, respectively.

The paper is organized as follows: In the next section we present the phase diagram of the quadrumerized SSM as obtained from boson operator mean-field theory. Then in the third section we use exact numerical diagonalization to study the effects of frustration in the model and relate the numerical results to the mean-field phase diagram. We concentrate on the standard SSM in the fourth chapter and provide evidence for a VBC intermediate phase. A summary and conclusions are given in the final section.

\section{BOSON OPERATOR APPROACH}

We first review the numerical results obtained along the unfrustrated lines in the parameter space of the Hamiltonian (1). For the $1 / 5$-th depleted square lattice $(J=0)$ there exists a plaquette RVB-like phase (PRVB) at small $J^{\prime} / K<0.94$, and a dimer RVB-like phase (DRVB) at large $J^{\prime} / K>1.67$, with an intermediate long-range ordered antiferromagnetic phase. [4] The classical configuration corresponding to this order is depicted by the arrows in Fig. 1.

The plaquette square lattice $\left(J^{\prime}=0\right)$ is unfrustrated as well. Using stochastic series expansion quantum Monte Carlo simulations a quantum critical point is found at $(J / K)_{c} \approx 0.55$, separating a PRVB spin liquid for $J / K<$ $(J / K)_{c}$ from a gapless Néel-ordered phase. 13] This value agrees well with results from perturbation expansions. 14 .

While quantum Monte Carlo proves powerful for studying the unfrustrated limits of the Hamiltonian (1), due to the sign problem other methods are needed, once frustration is present. In order to study possible instabilities of the spin liquid phases, we use standard boson operator mean-field theory, which is known to work on a qualitative level even for large frustration. Our analytical results will also be substantiated by the numerical approach of the following sections.

\section{A. Dimer-boson approach}

Consider first the DRVB regime $J^{\prime} \gg J, K$ where the dimer-boson technique can be applied. [15] In this representation, the spin- $1 / 2$ degrees of freedom on each $J^{\prime}$ dimer are expressed by bosonic bond operators,

$$
\begin{aligned}
|s\rangle & =s^{\dagger}|0\rangle=|0,0\rangle, \\
\left|t_{+}\right\rangle & =t_{+}^{\dagger}|0\rangle=|1,-1\rangle, \\
\left|t_{0}\right\rangle & =t_{0}^{\dagger}|0\rangle=|1,0\rangle, \\
\left|t_{-}\right\rangle & =t_{-}^{\dagger}|0\rangle=|1,+1\rangle,
\end{aligned}
$$

where the $\left|S, S^{z}\right\rangle$ denote the states on a given dimer. From the action of the spin operators $\mathbf{S}_{i}, i=1,2$ (denoting the two sites of a dimer) on these states the representation of the spin operators can be deduced

$$
\begin{aligned}
& S_{i}^{z}=\frac{1}{2}\left(t_{+}^{\dagger} t_{+}-t_{-}^{\dagger} t_{-}\right)-\frac{(-1)^{i}}{2}\left(t_{0}^{\dagger} s+s^{\dagger} t_{0}\right), \\
& S_{i}^{ \pm}=\frac{1}{\sqrt{2}}\left(t_{ \pm}^{\dagger} t_{0}+t_{0}^{\dagger} t_{\mp}\right) \pm \frac{(-1)^{i}}{\sqrt{2}}\left(t_{ \pm}^{\dagger} s-s^{\dagger} t_{\mp}\right) .
\end{aligned}
$$

The spin commutation relations for $\mathbf{S}_{i}, i=1,2$ are obtained when the bond operators obey bosonic statistics. Furthermore, the number of physical states available on each dimer specifies a hard-core constraint for the bosons on each dimer $\mu$,

$$
s_{\mu}^{\dagger} s_{\mu}+t_{\mu,+}^{\dagger} t_{\mu,+}+t_{\mu, 0}^{\dagger} t_{\mu, 0}+t_{\mu,-}^{\dagger} t_{\mu,-}=1 .
$$

Taking the interdimer couplings $J$ and $K$ into account and using Eq. (3), the Hamiltonian (1) is mapped onto an equivalent bosonic Hamiltonian, $H^{D}=H_{0}^{D}+H_{I}^{D}$, containing quadratic, diagonal terms

$H_{0}^{D}=J^{\prime} \sum_{\mu}-\frac{3}{4} s_{\mu}^{\dagger} s_{\mu}+\frac{1}{4}\left(t_{\mu+}^{\dagger} t_{\mu,+}+t_{\mu, 0}^{\dagger} t_{\mu, 0}+t_{\mu,-}^{\dagger} t_{\mu,-}\right)$,

and quartic terms, $H_{I}^{D}$, describing the interdimer scattering. The square lattice of dimers, with two dimers per unit cell, is found to reduce to a square lattice with a single site per unit cell in the bosonic representation. Here, we first imagine rotating half of the $J^{\prime}$-dimers clockwise, so that all dimers align along the $(1,-1)$-direction (cf. Fig. $1)$. Then we take the centers of the dimers as the sites and use a coordinate system where $x_{D}$ is along the original $(1,-1)$-direction and $y_{D}$ along $(1,1)$. To proceed, we need to implement the constraint (4) by means of a HolsteinPrimakoff representation, [16]

$$
s^{\dagger}=s=\sqrt{1-t_{+}^{\dagger} t_{+}-t_{0}^{\dagger} t_{0}-t_{-}^{\dagger} t_{-}},
$$

and then decouple the quartic $H_{I}^{D}$ via a linear approximation, similar to linear spin wave theory. The resulting total quadratic Hamiltonian, $\bar{H}^{D}$, is then diagonalized in momentum space using a generalized Bogoliubov transformation. 17] This approach is expected to work well inside the DRVB phase. A threefold degenerate spectrum of triplet excitations is obtained, consistent with unbroken $\mathrm{SU}(2)$ symmetry,

$$
\omega\left(\mathbf{k}_{D}\right)=\sqrt{J^{\prime}\left[J^{\prime}+(K-J)\left(\cos k_{x}-\cos k_{y}\right)\right]} .
$$

Here, the wave vector $\mathbf{k}_{D}=\left(k_{x}, k_{y}\right)_{D}$ is defined with respect to the dimer coordinate system $\left(x_{D}, y_{D}\right)$. The phase boundaries of the DRVB phase are obtained from the instabilities of the triplet excitation spectrum, i.e. by a vanishing spin gap at $\mathbf{k}_{D}=(\pi, 0)_{D}$, signaling the condensation of the corresponding bosons at this wave vector. 15] Mapping back onto the original square lattice, 
the corresponding magnetic order is obtained, characterizing the phase beyond the instability line $2(K-J) \geq J^{\prime}$. In fact, this magnetic order corresponds to the long-range order found for the $1 / 5$-th depleted square lattice, c.f. Fig. 1.

\section{B. Quadrumer-boson approach}

When the parameters in the Hamiltonian of Eq. (1) are close to another limiting case, $J, J^{\prime} \ll K$, a similar approach, the quadrumer-boson technique, can be applied. 18] The Hamiltonian of a single quadrumer, $H=K\left[\mathbf{S}_{1} \cdot \mathbf{S}_{2}+\mathbf{S}_{2} \cdot \mathbf{S}_{3}+\mathbf{S}_{3} \cdot \mathbf{S}_{4}+\mathbf{S}_{4} \cdot \mathbf{S}_{1}\right]$, can be expressed in terms of the total spin, $\mathbf{S}=\mathbf{S}_{1}+\mathbf{S}_{2}+\mathbf{S}_{3}+\mathbf{S}_{4}$, and the total subspin on each diagonal, $\mathbf{S}_{A}=\mathbf{S}_{1}+$ $\mathbf{S}_{3}$, and $\mathbf{S}_{B}=\mathbf{S}_{2}+\mathbf{S}_{4}$. The spectrum is given by $E\left(\left|S, S^{z}, S_{A}, S_{B}\right\rangle\right)=K / 2\left(S^{2}-S_{A}^{2}-S_{B}^{2}\right)$. The lowest lying triplet $\left\{\left|1, S^{z}, 1,1\right\rangle, S^{z}=0, \pm 1\right\}$ has a gap, $\Delta=K$, to the ground state $|0,0,1,1\rangle$. Since there is a further gap, $\Delta^{\prime}=K$, to the higher excitations, we attempt to obtain the instabilities of the PRVB phase by using a restricted quadrumer-boson method, omitting all the higher excitations on the quadrumers. Hence, the spin- $1 / 2$ degrees of freedom on each quadrumer are expressed by bosonic operators on the restricted Hilbert space,

$$
\begin{aligned}
|s\rangle & =s^{\dagger}|0\rangle=|0,0,1,1\rangle, \\
\left|t_{+}\right\rangle & =t_{+}^{\dagger}|0\rangle=|1,-1,1,1\rangle, \\
\left|t_{0}\right\rangle & =t_{0}^{\dagger}|0\rangle=|1,0,1,1\rangle, \\
\left|t_{-}\right\rangle & =t_{-}^{\dagger}|0\rangle=|1,+1,1,1\rangle .
\end{aligned}
$$

From the action of the spin operators $\mathbf{S}_{i}, i=1, \ldots, 4$ in the restricted Hilbert space, the following representation can be deduced [19]

$$
\begin{aligned}
& S_{i}^{z}=\frac{1}{4}\left(t_{+}^{\dagger} t_{+}-t_{-}^{\dagger} t_{-}\right)-\frac{(-1)^{i}}{\sqrt{6}}\left(t_{0}^{\dagger} s+s^{\dagger} t_{0}\right), \\
& S_{i}^{ \pm}=\frac{1}{2 \sqrt{2}}\left(t_{ \pm}^{\dagger} t_{0}+t_{0}^{\dagger} t_{\mp}\right) \pm \frac{(-1)^{i}}{\sqrt{3}}\left(t_{ \pm}^{\dagger} s-s^{\dagger} t_{\mp}\right) .
\end{aligned}
$$

In the restricted Hilbert space, the hard-core constraint (画) is now obeyed on each quadrumer. Expressing the Hamiltonian (1) in terms of the quadrumer-boson operators, a bosonic Hamiltonian, $H^{P}=H_{0}^{P}+H_{I}^{P}$, is obtained with a noninteracting diagonal part

$$
H_{0}^{P}=K \sum_{\mu}-2 s_{\mu}^{\dagger} s_{\mu}-\left(t_{\mu+}^{\dagger} t_{\mu,+}+t_{\mu, 0}^{\dagger} t_{\mu, 0}+t_{\mu,-}^{\dagger} t_{\mu,-}\right)
$$

and a quartic scattering part $H_{I}^{P}$. Here the sum extends over the square lattice of quadrumers formed by the $K$ bonds in Fig. 1. Following the decoupling procedure already used in the dimer-boson approach, the following threefold degenerate triplet excitation spectrum is obtained in the PRVB regime,

$$
\omega\left(\mathbf{k}_{P}\right)=\sqrt{K\left[K-\frac{2}{3}\left(2 J-J^{\prime}\right)\left(\cos k_{x}+\cos k_{y}\right)\right]} .
$$

The minimum of this spectrum is located at $\mathbf{k}_{P}=(\pi, \pi)_{P}$ when $J^{\prime}>2 J$, and the gap vanishes for $J^{\prime}>3 / 4(K+$ $2 J)$, corresponding again to the order depicted in Fig. 1. Furthermore, for $J^{\prime}<2 J$ the minimum is located at $\mathbf{k}_{P}^{\prime}=(0,0)_{P}$, and the gap is again closed for $J^{\prime}<$ $2 J-3 / 4 K$. In this regime the model becomes long-range Néel-ordered.

Upon comparing the ground state energies from the dimer- and the quadrumer-boson approach inside the common range of stability, we can obtain the direct first order transition line between the DRVB and PRVB spin liquid phases. 18.

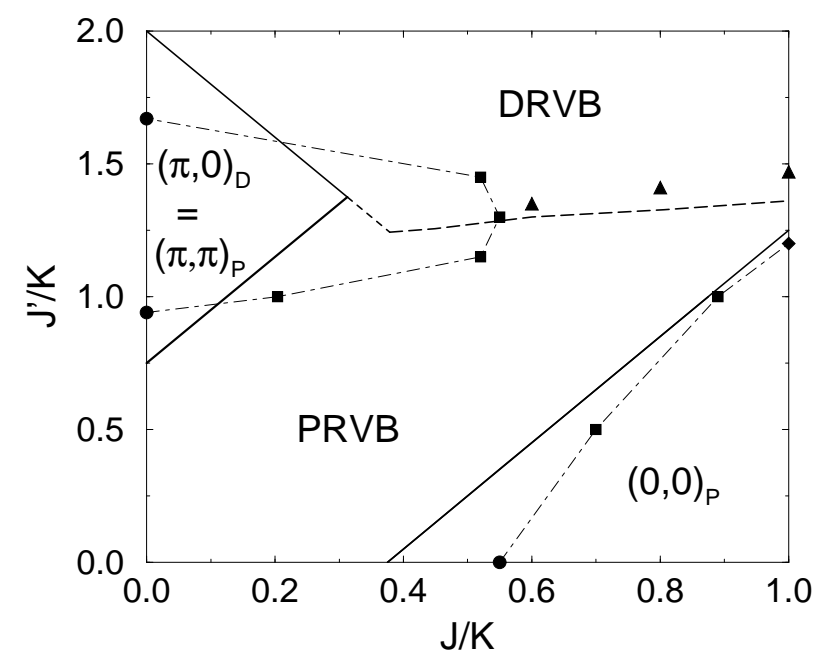

FIG. 2. Phase Diagram of the quadrumerized ShastrySutherland model. Ordered phases are characterized by the ordering wave vectors in the boson representations. Solid lines indicate second order transition lines, and the dashed line the first order transition. Also shown are points on the phase boundaries from quantum Monte Carlo (circles), [4,13] exact diagonalization (squares, and triangles), and series expansion (diamond). [12] Dashed-dotted lines are guides to the eye. The increment of the parameter scan in exact diagonalization was $\Delta(J / K)=0.1\left[\Delta\left(J^{\prime} / K\right)=0.2\right]$ for the $(\pi, \pi)_{P}\left[(0,0)_{P}\right]$ phase boundary.

The overall phase diagram is shown in Fig. 2. The spin liquid phases are characterized by the corresponding RVB-like state, while we label the long-range ordered phases by the ordering wave vectors in the boson operator approaches. Furthermore, solid lines represent second order phase transitions, whereas the dashed line indicates the first order transition line.

The Néel-ordered phase $(0,0)_{P}$ extends up to rather large frustration, with a largest extend of $\left(J^{\prime} / J\right)_{\max } \approx$ 1.2 along the Shastry-Sutherland line $(J=K)$. On the other hand, the largest extent of the $(\pi, \pi)_{P}$ phase, for $J^{\prime} / K \approx 1.3$ is bound by $(J / K)_{\max }<0.55$ from exact diagonalization. This difference can be traced back to 
the ratio of the number of frustrating couplings to the number the initial couplings, which is $1: 4$ when starting at $J^{\prime}=0$, but $2: 3$ upon starting at $J=0$.

Furthermore, from the phase diagram in Fig. 2, we find that the DRVB phase of the $1 / 5$-th depleted square lattice is adiabatically connected to the exact dimer VBS phase of the standard Shastry-Sutherland model (the dimer VBS state fails to be an exact eigenstate for $J \neq K)$. On the other hand, the DRVB phase is not adiabatically connected to the PRVB, as expected on topological grounds. 21] Hence, we find a first order phase transition separating the two spin liquid phases beyond the regime of the $(\pi, \pi)_{P}$ phase.

When turning to the case $J>K$, the phase diagram shown in Fig. 2 is obtained upon interchanging $J$ and $K$, due to the invariance of the Hamiltonian in Eq. (1) under the exchange $(J \leftrightarrow K)$. Furthermore, we label the plaquette RVB-like phase for $J>K$ by $\mathrm{PRVB}^{\prime}$, since now singlets are predominantly formed on a different set of quadrumers than in the PRVB phase.

\section{EXACT DIAGONALIZATION STUDIES}

We include in Fig. 2 the positions of quantum critical points along the unfrustrated lines, obtained by quantum Monte Carlo. [4,13] These compare rather well with the above mean-field theory. To extend the numerical analysis into the frustrated regime we have performed exact diagonalization studies on clusters with $N=8,16,32$ spins, using periodic boundary conditions, along various lines in the phase diagram. We determine the finite size values of the order parameter, $M^{\prime}$, defined by [20]

$$
M^{\prime 2}(N)=\frac{1}{N(N+2)}\left\langle\left(\sum_{i} \epsilon_{i} \mathbf{S}_{i}\right)^{2}\right\rangle .
$$

Here $\epsilon_{i}$ takes on the values \pm 1 at site $i$, according to the pattern in Fig. 1 for the $(\pi, \pi)_{P}$ phase or the standard Néel-order for the $(0,0)_{P}$ phase, respectively. Using the finite-size data we determine $M^{\prime}$ from the scaling law [20]

$$
M^{\prime}(N)=M^{\prime}+\frac{c_{1}}{N^{1 / 2}}+\frac{c_{2}}{N}
$$

For example, in Fig. 3 we show results obtained along the line $J^{\prime}=1.3$, where quantum Monte Carlo simulations at $J=0$ give a maximum moment of $M^{\prime} \approx 0.23$. [4] Within exact diagonalization we can reproduce this value, and furthermore observe a smooth decrease in $M^{\prime}$ upon increasing the frustration, up to a critical point at $J / K \approx 0.55$, where we enter into the spin liquid regime. Proceeding in a similar fashion we obtain the critical points depicted by squares in Fig. 2. Moreover, along the line $J^{\prime}=2 J$ no finite order parameter was obtained after finite size scaling.

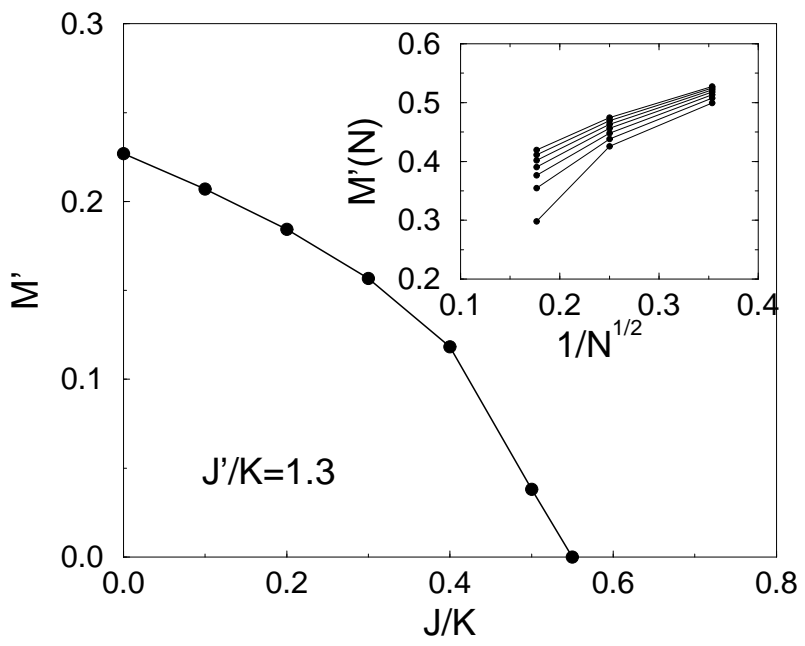

FIG. 3. Evolution of the order parameter of the $(\pi, \pi)_{P}$ phase upon increasing $J / K$ for $J^{\prime} / K=1.3$. The inset showns the finite size data obtained for $N=8,16,32$ at $J / K=$ $0,0.1, \ldots, 0.6$, (top to bottom).

The two spin liquid phases cannot be separated within exact diagonalization using space group symmetry. Namely, upon increasing $J^{\prime} / K$ for a constant $J / K$, the representation class of the ground state does not change. Nevertheless, from the approximate slopes of the ground state energy vs. $J^{\prime} / K$ at constant $J / K$ in the regions of small and large $J^{\prime} / K$ respectively, we estimated the first order transition points indicated by triangles in Fig 2.

Comparing these numerical results with the mean-field calculations in the last section, we conclude that the boson operator approach gives a good qualitative account of the phase diagram of the quadrumerized SSM. Namely, the characterization of the various phases and the location of the phase transition lines agree well with numerical results.

\section{SHASTRY-SUTHERLAND MODEL}

In the quadrumer-boson approach we find a finite window on the Shastry-Sutherland line $(J=K)$, where both plaquette spin liquid phases, $\mathrm{PRVB}$ and $\mathrm{PRVB}^{\prime}$, come arbitrary close to the $J=K$ line. This already indicates an intermediate phase in the standard SSM between the Néel-ordered phase and the dimer VBS phase. Similar conclusions were obtained in a field theoretical study of a generalized SSM, which does not break the symmetry needed by the dimer VBS state to be an exact eigenstate. 11] We now focus on this intermediate regime in the standard SSM.

There has recently been considerable interest in the nature of the intermediate phase. In the large spin, classical limit the system retains Néel-order for $J^{\prime} / J \leq 1$ and is helically ordered otherwise, with a twist between next 
nearest neighbour spins of $q=\arccos \left(-J / J^{\prime}\right)$. Using Schwinger boson mean-field theory, Albrecht and Mila predicted the existence of a helical phase separating the dimer VBS and the ordered phase also for the spin- $1 / 2$ case, in a range $1.1<J^{\prime} / J<1.65$. [8] Field-theoretical studies by Chung, Marston, and Sachdev for a generalized spin-S model with $\mathrm{Sp}(2 \mathrm{~N})$ symmetry suggest the helical order to occur in a larger range, $1.02<J^{\prime} / J<2.7$, at $S=1 / 2$. [10] Furthermore, this approach predicts a phase with plaquette order in the extreme quantum limit, $1 / S>5$. Indeed, using series expansions around the plaquette limit of Eq. (1), Koga and Kawakami found the intermediate phase in a range $1.16<J^{\prime} / J<1.48$ to be adiabatically connected to the PRVB phase. [9] However, extended series expansions by Weihong, Oitmaa, and Hamer lead to different conclusions. [12] They suggest the PRVB phase to become unstable before the Shastry-Sutherland line is reached and found a columnar dimer phase to be a possible candidate for the intermediate phase.

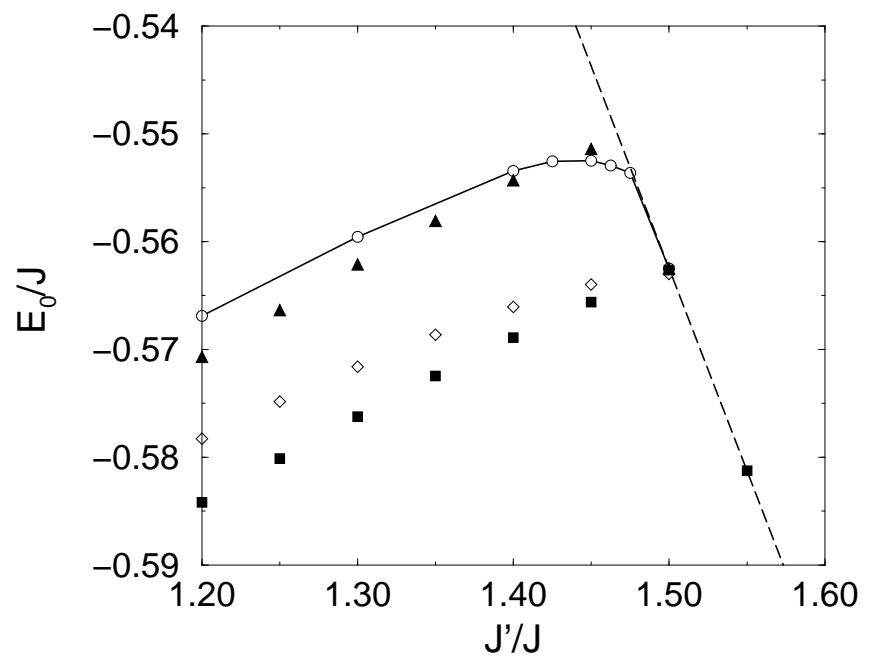

FIG. 4. Ground state energy per site of the Shastry-Sutherland model from exact diagonalization of clusters with $N=16$ (squares), 20 (triangles), 24 (diamonds), and 32 (circles) sites. The solid line is a guide to the eye, and the dashed line is the exact dimer VBS state energy.

Here, we perform exact diagonalization studies on clusters with up to 32 sites, significantly beyond the largest cluster sizes studied so far (24 sites in Ref. 221). In Fig. 4 we show the ground state energy of the SSM close to the transition into the dimer VBS state for various cluster sizes. The solid line shows our results for the 32 site cluster, whereas other sizes are represented by symbols. In addition, the energy of the dimer VBS state is shown by the dashed line. Due to the different symmetry of the various clusters, the ground state enery per site does not show a monotonous finite size dependence. Nevertheless, independent of the cluster size we find the system to be within the dimer VBS for $J^{\prime} / J>1.5$, consistent with the upper bound for the intermediate phase given by Koga and Kawakami, but significantly below the values from the Schwinger boson, and $\mathrm{Sp}(2 \mathrm{~N})$ theory. From finite size analysis of the Néel-order parameter, we estimate a upper bound for the ordered phase of $\left(J^{\prime} / J\right)_{\max }<1.4$, consistent with the series expansion value $\left(J^{\prime} / J\right)_{\max }=1.2 \pm 0.1$ [12. More interestingly, for the largest cluster we find a characteristic change in the curvature of the ground state energy, well before entering the dimer VBS. Hence, we conclude that characteristic features of the intermediate phase could be retrieved from clusters with $N=32$ sites in a range $1.425<J^{\prime} / J<1.475$.

In this regime we find the spin-spin, or 2-point correlation function to decrease rapidly with distance, indicating the absence of antiferromagnetic order.

\begin{tabular}{|c|c|c|c|}
\hline$(k, l)$ & $C^{4}(0,16 ; k, l) \|$ & $(k, l)$ & $C^{4}(0,16 ; k, l)$ \\
\hline 25,26 & 0.0213 & 20,26 & 0.0140 \\
\hline 29,30 & 0.0222 & 21,27 & 0.0180 \\
\hline 31,9 & 0.0788 & 22,1 & 0.0274 \\
\hline 2,3 & 0.0825 & 17,23 & 0.0152 \\
\hline 6,7 & 0.0222 & 18,24 & 0.0148 \\
\hline 12,13 & 0.0209 & 8,14 & 0.0175 \\
\hline 8,28 & 0.0140 & 28,15 & 0.0239 \\
\hline 14,15 & 0.0144 & 29,31 & 0.0274 \\
\hline 21,22 & 0.0140 & 9,30 & 0.0152 \\
\hline 1,27 & 0.0153 & 4,10 & 0.0239 \\
\hline 4,5 & 0.0138 & 5,11 & 0.0175 \\
\hline 10,11 & 0.0160 & 6,12 & 0.0148 \\
\hline 17,18 & 0.0140 & 7,13 & 0.0180 \\
\hline 23,24 & 0.0214 & 19,25 & 0.0140 \\
\hline 19,20 & 0.0223 & 13,20 & -0.0124 \\
\hline 24,25 & -0.0107 & 14,21 & -0.0143 \\
\hline 28,29 & -0.0104 & 15,22 & -0.0207 \\
\hline 15,31 & -0.0007 & 10,17 & -0.0019 \\
\hline 1,2 & -0.0007 & 11,18 & -0.0135 \\
\hline 5,6 & -0.0137 & 27,4 & -0.0177 \\
\hline 11,12 & -0.0107 & 1,5 & -0.0177 \\
\hline 30,4 & -0.0104 & 2,6 & -0.0019 \\
\hline 9,10 & -0.0292 & 3,7 & -0.0207 \\
\hline 3,23 & -0.0292 & 23,8 & -0.0103 \\
\hline 7,8 & -0.0137 & 24,28 & -0.0164 \\
\hline 13,14 & -0.0163 & 25,29 & -0.0142 \\
\hline 20,21 & -0.0125 & 26,30 & -0.0135 \\
\hline 26,27 & -0.0163 & 12,19 & -0.0138 \\
\hline 18,19 & -0.0125 & & \\
\hline
\end{tabular}

TABLE I. Dimer-dimer correlations $C^{4}(1,2 ; k, l)$ in the ground state of the Shastry-Sutherland model on the 32 sites cluster at $J^{\prime} / J=1.45$. The labeling of the sites is shown in Fig. 5 . 
In order to test against the various proposed ground states, we measure the dimer-dimer, or 4-point correlation functions,

$$
C^{4}(i, j, ; k, l)=\left\langle\mathbf{S}_{i} \cdot \mathbf{S}_{j} \mathbf{S}_{k} \cdot \mathbf{S}_{l}\right\rangle-\left\langle\mathbf{S}_{i} \cdot \mathbf{S}_{j}\right\rangle\left\langle\mathbf{S}_{k} \cdot \mathbf{S}_{l}\right\rangle,
$$

on the $N=32$ lattice in the above interaction range. In particular, we fix $(i, j)=(0,16)$ and extend $(k, l)$ over all inequivalent $J$ bonds. The values obtained in the ground state for $J^{\prime} / J=1.45$ are displayed in Table I, and illustrated in Fig. 5, which also shows the labeling of the sites.

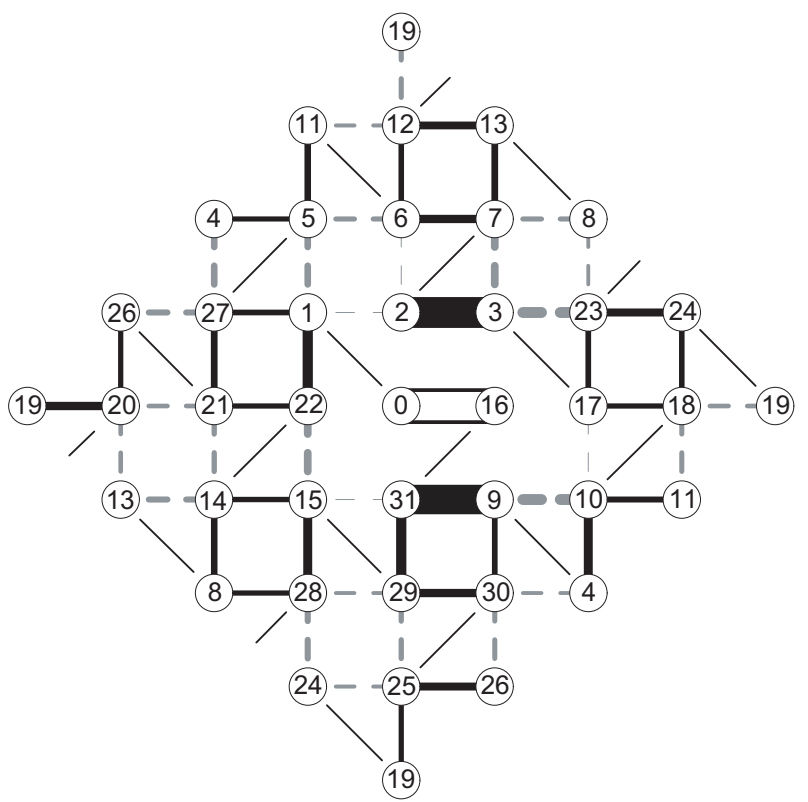

FIG. 5. Dimer-dimer correlations in the ground state of the Shastry-Sutherland model on the 32 sites cluster at $J^{\prime} / J=1.45$. The reference bond is the bond $(0,16)$. Positive (negative) correlations are drawn as full (dashed) lines. The thickness of a line is proportional to the strength of the correlation. Short diagonal lines indicate the position of the $J^{\prime}$-dimer bonds.

We obtain a clear signal in the dimer-dimer correlations that extends throughout the whole cluster, with a finite asymptotic value approximately reached for the larger dimer-dimer distances on the cluster. In the spatial distribution we furthermore observe periodic oscillations, which reflect an underlying order of quadrumer-singlets, formed predominately on void squares (i.e. those squares not containing a diagonal bond).

For the SSM there are two equivalent configurations with quadrumer-singlet coverings residing on the two different subsets of void squares (formed by the $J$ or $K$ bonds in Fig. 1, respectively). Hence, if the plaquettelike order in the 4-spin correlation function survives quantum fluctuations, indicative of a plaquette VBC, a two-fold degenerate ground state manifold will emerge in the thermodynamic limit. On a finite lattice this degen- eracy is lifted, but a low-lying singlet state well inside the triplet gap and only slightly above the ground state energy is expected. We can obtain the quantum numbers of this singlet state from the following symmetry considerations: The Shastry-Sutherland lattice has a $44 \mathrm{~mm}$ space group symmetry, 23] and the ground state of the 32 site cluster has momentum $\mathbf{k}=(0,0)$, and is invariant under the $\pi / 2$ rotations about the center of any void square (i.e. s-wave like). Furthermore, the two equivalent configurations of quadrumer-singlet coverings are related by the reflections about either diagonal dimer axes, but are invariant under the $\pi / 2$ rotations and lattice translations. Namely, they both reside already inside a single unit cell of the Shastry-Sutherland lattice.

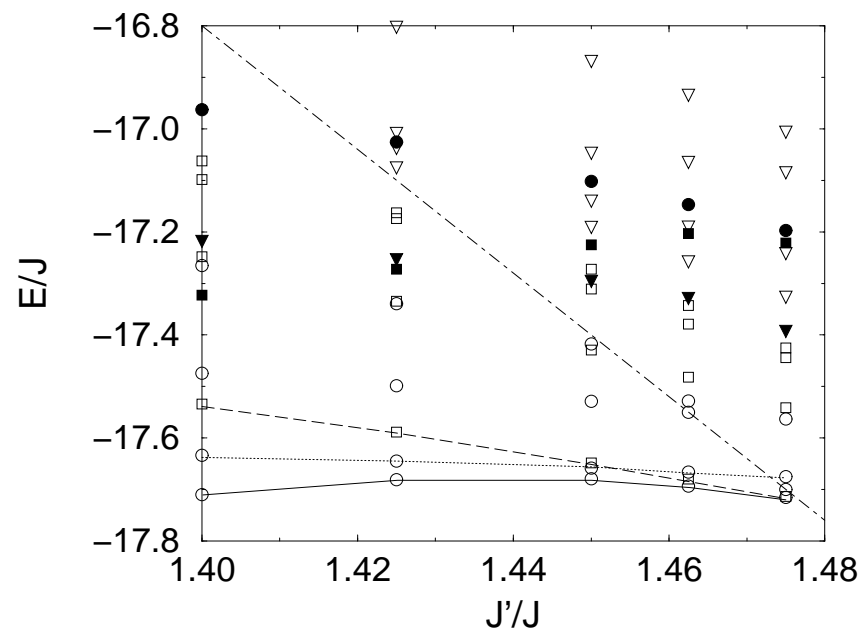

FIG. 6. Ground state energy, and low-lying singlet and triplet excitations in the $\mathbf{k}=(0,0)$ sector of the Shastry-Sutherland model on the 32 sites cluster. Open symbols represent singlet states, full symbols triplet states. Circles denote states with eigenvalues $R_{\pi / 2}=1$ (s-wave), squares denote $R_{\pi / 2}=-1$ (d-wave), and triangles $R_{\pi / 2}= \pm i$ (two-fold degenerate). The solid line is a guide to the eye for the ground state, the dotted to the lowest excited s-wave state, and the dashed line the lowest d-wave state. The Dashed-dotted line is the exact dimer VBS state energy.

Hence, a low-lying s-wave symmetric singlet state with momentum $(0,0)$ is expected to be included in the spectrum of the 32 site cluster in the regime of the plaquette VBS phase. This state should furthermore show similar dimer-dimer correlations as the absolute ground state.

In Fig. 6 we plot the ground state energy along with those of the lowest excited singlet and triplet states for the 32 site cluster in the zero momentum sector. We specify the transformation properties of the various states under the $\pi / 2$ rotation by the eigenvalue $R_{\pi / 2}=1$ ( $\mathrm{s}$ wave), -1 (d-wave), or $\pm i$ (two-fold degenerate). See the caption of Fig. 6 for a detailed account on the various used symbols. In the regime where we expect evidence for an intermediate state we indeed find various low-lying singlet states well inside the rather large triplet 
gap. Moreover, there are two singlet states with energies rather close to the ground state energy, one being a s-wave, and the other a d-wave state with respect to the $\pi / 2$ rotations. We furthermore calculated the dimerdimer correlations for both states and find for the s-wave state a similar signal as for the absolute ground state. On the other hand, the d-wave state does not show any pattern in the dimer-dimer correlations. This state does not seem relevant for the ground state of the system in the thermodynamic limit. Presumably it is related to low-lying excitations in the dimer VBS phase. 2223]

From the above we conclude that the low-lying s-wave singlet state, having the right quantum numbers and dimer-dimer correlation, will become degenerate with the ground state upon increasing the cluster size to the infinite lattice. Both states then form the two-fold degenerate ground state manifold of a plaquette $\mathrm{VBC}$ in the thermodynamic limit. In Fig. 7 a pictorial description of this ground state manifold is given.
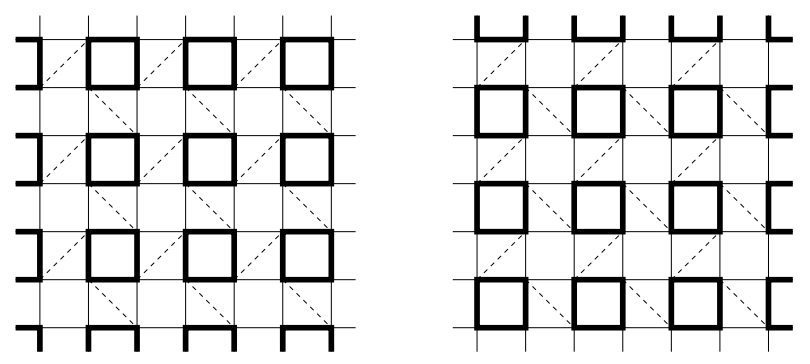

FIG. 7. Pictorial illustration of the variational ground state manifold of the Shastry-Sutherland model in the plaquette VBC phase. Thick lines indicate four spins involved in a quadrumer-singlet.

Furthermore, this ground state manifold is invariant with respect to lattice translations and the $\pi / 2$ rotations about the centers of the void squares, but each state spontaneously breaks the reflection symmetry about the diagonal axes along the dimer directions.

From our numerical results we can also exclude a columnar dimer state, which would be four-fold degenerate and furthermore show a different pattern in the dimer-dimer correlations than depicted in Fig. 5. 26]

Further evidence for the relevance of the plaquette VBC in the intermediate regime of the SSM can be drawn from analogous results of recent studies on the spin-1/2 Heisenberg model on the checkerboard lattice. 24,25] In this model, diagonal bonds are again organized on a square lattice in a pattern as to leave half of the squares void. Namely, the underlying lattice is obtained by adding in an additional diagonal bond on each square on which a dimer bond is located in Fig. 1. Also in the checkerboard lattice quadrumer-singlets form on the void squares, resulting in a two-fold degenerate plaquette VBC ground state manifold with broken space group symmetry. The structure leading to frustration is rather similar in both models, and the system tries to minimize frustration by forming plaquette valence bonds on the void squares. Due to the homogeneous axial couplings, this can be accomplished only upon spontaneously breaking the symmetry inherited from the underlying lattice.

\section{CONCLUSIONS}

In conclusion, we have studied the phase diagram of the quadrumerized Shastry-Sutherland model. Using bondoperator methods and exact numerical diagonalization its phase diagram was established, which links the various results available for special limiting cases of the model. The antiferromagnetically ordered phase of the $1 / 5$-th depleted square lattice model is destroyed by modest frustration, whereas the Néel-ordered phase extends up to rather large frustration. There is a first order transition line separating the different spin liquid phases, PRVB and DRVB, beyond the ordered phase. Furthermore, the DRVB is adiabatically connected to the dimer VBS phase of the SSM.

For the standard SSM there exists a finite region around $J^{\prime} / J=1.45$ where the system becomes a plaquette VBC with spontaneously broken space group symmetry, and a two-fold degenerate ground state manifold. Perturbing away from the Shastry-Sutherland line, i.e. for $J \neq K$, the symmetry is broken explicitly, and the system favors a unique ground state, namely the PRVB for $J<K$, and the PRVB' for $J>K$. Furthermore, upon varying $J^{\prime}$ along the Shastry-Sutherland line, a first order transition leads to the dimer VBS and a second order transition to the Néel-ordered phase. Within our analytical and numerical studies we did not find indications for further phases in the (quadrumerized) SSM.

Stabilization of a plaquette VBC phase in both the Shastry-Sutherland and the checkerboard lattice model agrees with the generic structure of the underlying frustrated lattice. However, in the SSM the VBC is unstable towards the dimer VBS upon increasing the diagonal coupling. For the checkerboard lattice model the range of the VBC phase is still unkown, and remains for further studies.

We acknowledge fruitful discussions with Stephan Haas, Andreas Honecker, and Bruce Normand. This work has been financially supported by the Swiss Nationalfonds.

Note added.- After completion of this work Akihisa Koga, and Norio Kawakami pointed us to Ref. [27, where the phase diagram of the Hamiltonian in Eq. (1) was studied using series expansion methods. Their results are in perfect agreement with our calculations in sections II and III. However in [27] the VBC nature of the intermediate phase in the Shastry-Sutherland model was not noticed. 
[1] For a recent review, see e.g.: C. Lhuillier, and G. Misguich, cond-mat/0109146.

[2] K. Ueda, H. Kontani, M. Sigrist, and P. A. Lee, Phys. Rev. Lett. 76, 1932 (1996).

[3] N. Katoh and M. Imada, J. Phys. Soc. Jpn. 64, 4105 (1995).

[4] M. Troyer, H. Kontani, and K. Ueda, Phys. Rev. Lett. 76, 3822 (1996); M. Troyer, M. Imada, and K. Ueda, J. Phys. Soc. Jpn. 66, 2957 (1997).

[5] H. Kageyama, K. Yoshimura, R. Stern, N. V. Mushnikov, K. Onizuka, M. Kato, K. Kosuge, C. P. Slichter, T. Goto, and Y. Ueda, Phys. Rev. Lett. 82, 3166 (1999).

[6] S. Miyahara and K. Ueda, Phys. Rev. Lett. 82, 3701 (1999).

[7] B.S. Shastry and B. Sutherland, Physica 108B, 1069 (1981).

[8] M. Albrecht and F. Mila, Europhys. Lett. 34, 145 (1996).

[9] A. Koga and N. Kawakami, Phys. Rev. Lett 84, 4461 (2000).

[10] C.H. Chung, J.B. Marston, and S. Sachdev, Phys. Rev. B 64, 134407 (2001).

[11] D. Carpentier, and L. Balents, Phys. Rev. B 6524427 (2002).

[12] Weihong Zheng, J. Oitmaa, and C. J. Hamer, Phys. Rev. B 6514408 (2002).

[13] S. Wessel, unpublished.

[14] Y. Zukumoto, and A. Oguchi, J. Phys. Soc. Jpn. 67, 2205 (1998)

[15] S. Sachdev and R. N. Bhatt, Phys. Rev. B 41, 9323 (1990).

[16] A. V. Chubukov and Th. Jolicoeur, Phys. Rev. B 44 12050 (1991).

[17] Th. Jolicoeur and J. C. Guillou, Phys. Rev. B 40, 2727 (1989).

[18] O.A. Starykh, M.E. Zhitomirsky, D. I. Khomskii, R. R. P. Singh, and K. Ueda, Phys. Rev. Lett. 77, 2558 (1996).

[19] Keeping all excited states and the corresponding bosons, 26 more bilinear terms appear for each spin operator.

[20] M. Albrecht, F. Mila, and D. Poilblanc, Phys. Rev. B 54 15856 (1996).

[21] N. E. Bonesteel, Phys. Rev. B 40, 8954 (1989).

[22] K. Totsuka, S. Miyahara, and K. Ueda, Phys. Rev. Lett. 86520 (2001).

[23] C. Knetter, A. Bühler, E. Müller-Hartmann, and G. S. Uhrig, Phys. Rev. Lett. 85, 3958 (2000).

[24] J.-B. Fouet, M. Mambrini, P. Sindzingre, and C. Lhuillier, cond-mat/0108070.

[25] W. Brenig and A. Honecker, Phys. Rev. B 65, 140407(R) (2002).

[26] P. W. Leung and N. Lam, Phys. Rev. B 53, 2213 (1996).

[27] Y. Takushime, A. Koga, and N. Kawakami, J. Phys. Soc. Jpn. 70, 1369 (2001). 